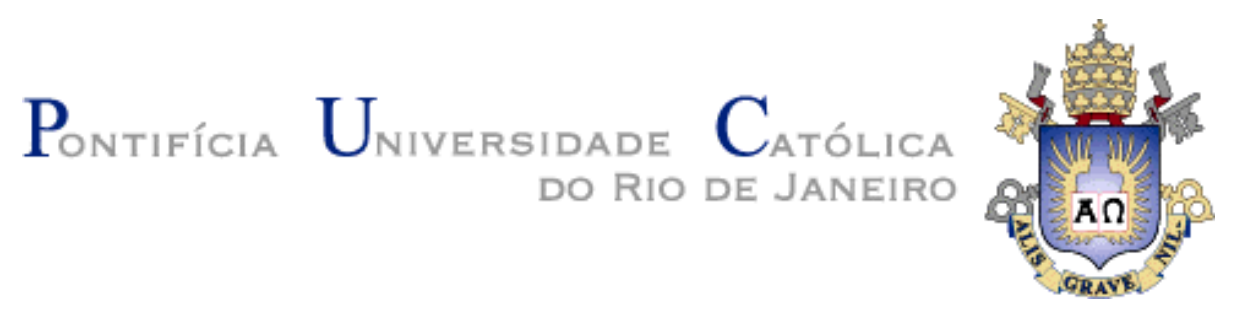

Fabio Seixas Marques

\title{
Um Processo Baseado em MDA para a Especialização de Mecanismos de Persistência
}

Dissertação apresentada como requisito parcial para obtenção do título de Mestre pelo Programa de PósGraduação em Informática da PUC-Rio.

Orientador: Carlos José Pereira de Lucena Co-Orientador: Ivan Mathias Filho 


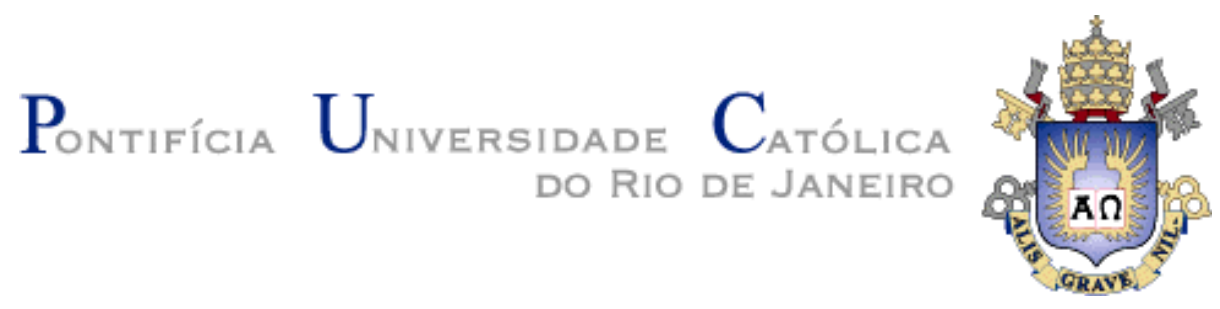

Fabio Seixas Marques

\section{Um Processo Baseado em MDA para a Especialização de Mecanismos de Persistência}

Dissertação apresentada como requisito parcial para obtenção do título de Mestre pelo Programa de PósGraduação em Informática da PUC-Rio. Aprovada pela Comissão Examinadora abaixo assinada.

Prof. Carlos José Pereira de Lucena Orientador

Departamento de Informática - PUC-Rio

Prof. Ivan Mathias Filho Co-Orientador Departamento de Informática - PUC-Rio

Prof. Arndt von Staa Departamento de Informática - PUC-Rio

Prof. Ricardo Choren Noya Seção de Engenharia de Computação - IME

Prof. José Eugênio Leal Coordenador Setorial do Centro Técnico Científico - PUC-Rio 
Todos os direitos reservados. É proibida a reprodução total ou parcial do trabalho sem autorização da universidade, do autor e do orientador.

Fabio Seixas Marques

Graduou-se em Bacharelado em Informática pela Pontifícia Universidade Católica do Rio de Janeiro no ano de 2002. É funcionário da SAU (Sistemas de Administração Universitária) da PUC-Rio.

Ficha Catalográfica

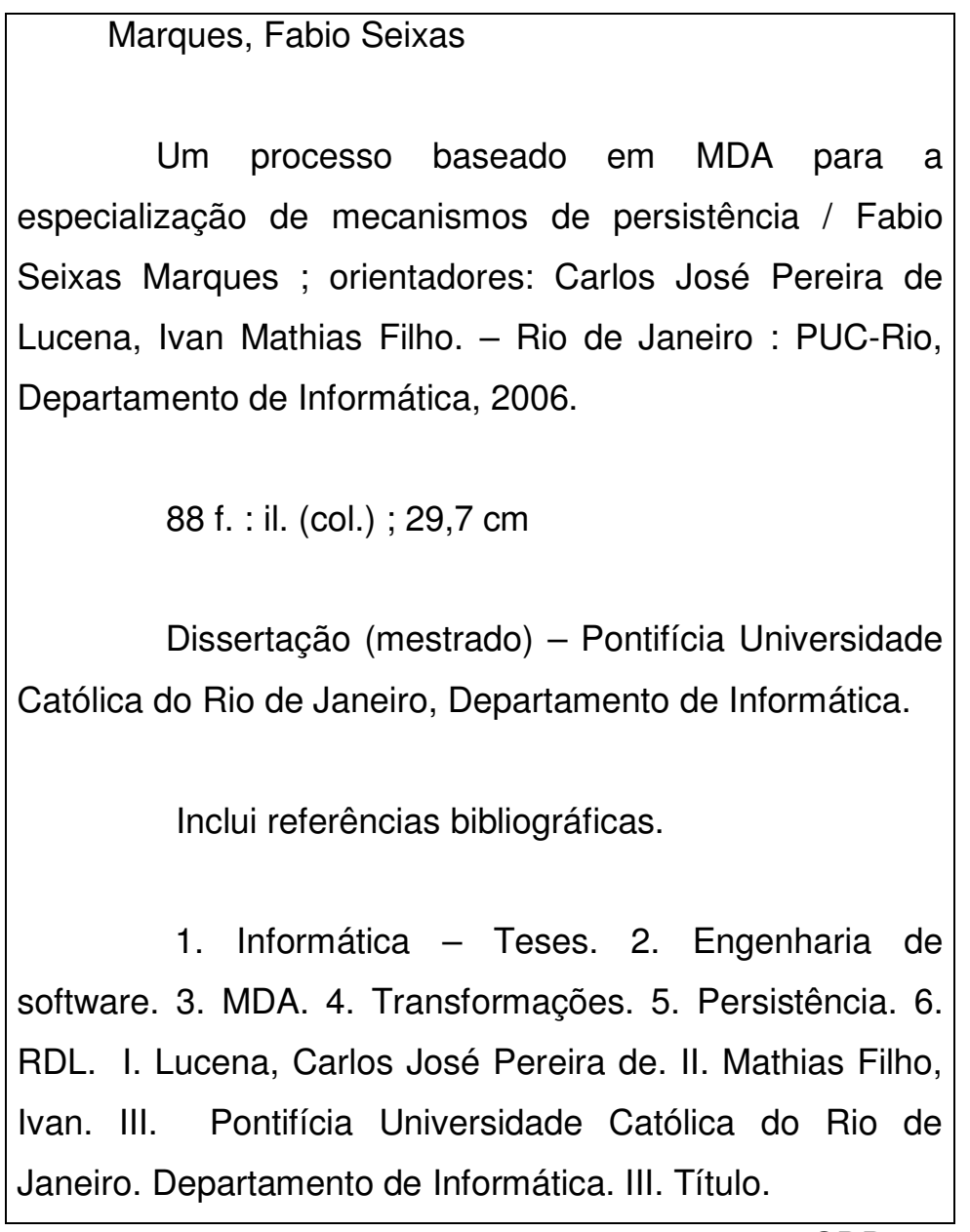

CDD: 004 
Para minha noiva, Ana Carina, meus pais, Antonio e Maria Lucia, e meu irmão, Junior, por todo apoio e amor nessa longa jornada. 


\section{Agradecimentos}

A minha noiva, Ana Carina Barros Queiroz, por todo apoio, amor e carinho incondicional durante todos esses anos de dedicação. Espero um dia conseguir retribuir.

Aos meus pais, Antonio e Maria Lucia, que sempre fizeram de tudo por mim e me criaram com tanto amor e carinho.

Ao meu irmão, Junior, por todo companheirismo e ajuda desde que éramos crianças.

Aos meus avos, por todos os lanchinhos feitos com tanto amor.

Ao meu orientador, Carlos José Pereira de Lucena, pela confiança e pelo apoio que me deu durante todo este longo caminho.

Ao meu co-orientador, Ivan Mathias Filho, por toda a paciência e apoio desde o meu projeto final de graduação. Guardarei para sempre tudo o que fez por mim.

Aos meus colegas de trabalho, por toda a ajuda e apoio nos momentos mais difíceis.

A Déborah e Emanuelle, por sempre conseguirem dar um jeito nos meus problemas. 


\section{Resumo}

Marques, Fabio Seixas; Lucena, Carlos José Pereira de; Mathias Filho, Ivan. Um Processo Baseado em MDA para a Especialização de Mecanismos de Persistência. Rio de Janeiro, 2006. 88p. Dissertação de Mestrado - Departamento de Informática, Pontifícia Universidade Católica do Rio de Janeiro.

Com as constantes mudanças nos requisitos funcionais e não-funcionais do domínio das aplicações, há uma grande necessidade dos softwares se adaptarem a essas mudanças. Devido a isso, o processo proposto neste trabalho tem o intuito de automatizar algumas partes do desenvolvimento de aplicações, minimizando o trabalho manual necessário para adaptar os sistemas de software às constantes necessidades de mudanças determinadas pela realidade do mundo dos negócios. A abordagem Model Driven Architecture (MDA), definida pela OMG, foi utilizada como base para este processo por ter como objetivo principal a transformação de modelos; ou seja, gerar, a partir de um modelo independente de plataforma, um ou mais modelos específicos para uma plataforma. O processo proposto é dividido de acordo com as etapas da MDA, tendo como objetivo auxiliar nas etapas de geração de modelos e na adaptação de frameworks de persistência. Foi utilizado no processo em questão, o conceito de base de conhecimento, tendo como objetivo principal mapear as informações contidas no modelo com as regras de transformações a serem realizadas no mesmo. Essas regras são responsáveis pela definição das modificações a serem realizadas nos modelos independentes de plataforma com o objetivo de incluir um determinado framework de persistência em uma aplicação. A linguagem RDL foi utilizada para formalizar as regras de transformações utilizadas no processo, facilitando o desenvolvimento e a manutenção das mesmas. O processo tem como resultado um modelo específico para uma plataforma, contendo ainda um framework de persistência adaptado à arquitetura utilizada. Alguns arquivos de configuração também são gerados com o intuito de minimizar o trabalho manual.

\section{Palavras-chave}

Engenharia de Software; MDA; Transformações; Persistência; RDL 


\section{Abstract}

Marques, Fabio Seixas; Lucena, Carlos José Pereira de; Mathias Filho, Ivan. A Process Based on MDA for Specialization of Persistence Mechanisms. Rio de Janeiro, 2006. 88p. Dissertation - Departamento de Informática, Pontifícia Universidade Católica do Rio de Janeiro.

According to the changes on functional and non-functional requirements on application field, there is a great need for softwares to adapt themselves to these changes. Due to it, the process involved on this work has the purpose of automate some parts of applications' developments, reducing the manual work needed to adapt the software systems to the need of constant changes determined by business world's reality. The approach Model Driven Architecture (MDA), defined by OMG, was used as base for this process, having as main goal the transformation of models; i mean, generate from an platform independent model, one or more platform specific models. The process suggested is divided according to the MDA steps, having as a goal the support of generate models steps and on the adaptation of Persistence Frameworks. It was used on the suggested process, the concept of base of knowledge, having as main goal map the informations held within the model with the transformation rules to be done on these models. These rules are responsible by the definition of changes to be done on platform-independent models aiming to include a specific Persistence Framework in an application. The language RDL was used to formalize the transformation rules used in the process, making the development and maintenance of themselves easy the process has as a result a platform-specific model, also a Persistence Framework adapted to the architecture used. Some configuration files also are generated, intending to reduce the manual work.

\section{Keywords}

Software Engineering; MDA; Transformations; Persistence; RDL 


\section{Sumário}

1 Introdução 13

1.1. Desenvolvimento de Software 13

1.2. Generative Programming 13

1.3. Desenvolvimento de Software Utilizando MDA (Model Driven $\begin{array}{ll}\text { Architecture) } & 14\end{array}$

1.4. O Objetivo da Dissertação e o Resumo da Solução Proposta 15

1.5. Lista das Contribuições da Dissertação 16

1.6. Estrutura da Dissertação 17

2 Trabalhos Relacionados $\quad 18$

$\begin{array}{ll}\text { 2.1. Odyssey-MDA } & 18\end{array}$

2.2. UML Model Transformation Tool (UMT) 20

2.3. AppFuse 23

2.4. Model in Action (Mia) 24

$\begin{array}{ll}\text { 2.5. Metastorage } & 27\end{array}$

3 Tecnologias Relacionadas $\quad 31$

3.1. Model Driven Architecture (MDA) 31

3.1.1. Introdução 31

3.1.2. CIM (Compution Independent Model) 32

3.1.3. PIM (Plataform Independent Model) 32

3.1.4. PSM (Plataform Specific Model) 33

3.1.5. Marcações 33

3.1.6. Transformação do PIM em PSM 33

3.1.7. Geração de Código 34

3.2. XML Metadata Interchange (XMI) 34

3.3. Reuse Description Language (RDL) 35

3.4. Frameworks de Persistência 37

3.4.1. Castor 38

$\begin{array}{ll}\text { 3.4.2. Hibernate } & 39\end{array}$

3.4.3. iBatis 40 
4 Processo de Transformação 43

4.1. Criação do Modelo PIM 46

4.2. Configuração do Modelo PIM (Marcações) 46

4.3. Configuração da Base de Conhecimento 47

4.4. Execução das Regras de Transformações 49

4.5. Criação do Modelo PSM

4.6. Considerações Finais 56

5 Estudos de Caso $\quad 58$

5.1. Sistema de Sincronização dos Dados Acadêmicos do SAU e do $\begin{array}{ll}\text { AulaNet } & 58\end{array}$

5.2. Site de Leilão Virtual $\quad 64$

6 Ferramenta para a Especialização de Mecanismos de Persistência 71

6.1. Introdução

6.2. Manutenção da Base de Conhecimento 72

6.3. Execução do Processo $\quad 76$

7 Considerações Finais $\quad 82$

7.1. Conclusão

7.2. Principais Contribuições

7.3. Trabalhos Futuros $\quad 84$

8 Bibliografia $\quad 86$ 


\section{Lista de figuras}

Figura 1 - Exemplos de Generative Programming [Iseger05] 14

Figura 2 - Visão geral do Model Driven Architecture [MDA] 15

Figura 3 - Cenário de utilização da Odyssey-MDA [Odyssey-MDA05] 18

Figura 4 - Exemplo de um arquivo XML contendo as regras de

transformações [Odyssey-MDA05] 19

Figura 5 - Mecanismos genéricos de transformação (built-ins)

[Odyssey-MDA05] 20

Figura 6 - Arquitetura da Ferramenta UMT (Independente de Plataforma)

[UMT]

Figura 7 - Arquitetura da Ferramenta UMT (Específica para uma

Plataforma) [UMT]

Figura 8 - Exemplo de um arquivo XSLT contendo as regras de

transformação [UMT]

Figura 9 - Exemplo de configuração do Appfuse, para a geração de uma aplicação [AppFuse] 23

Figura 10 - Visão Geral da Ferramenta Model in Action [MIA] 25

Figura 11 - Exemplo de criação de um serviço, utilizando a parte gráfica da ferramenta Model in Action [MIA]

Figura 12 - Exemplo da criação de regras de transformação, sem utilizar a parte gráfica da ferramenta MIA [MIA] 26

Figura 13 - Arquitetura da Ferramenta Model in Action [MIA]

Figura 14 - Exemplo de criação de uma classe e seus atributos e métodos utilizando XML [Metastorage]

Figura 15 - Exemplo da criação de um report [Metastorage] 30

Figura 16 - Model Driven Architecture 32

Figura 17 - Transformação do Modelo PIM no Modelo PSM [MDA] 34

Figura 18 - Estrutura de um Script RDL [Oliveira05] 36

Figura 19 - Exemplo de um Modelo Simples 36

$\begin{array}{ll}\text { Figura } 20 \text { - Exemplo de um Script RDL } & 37\end{array}$

Figura 21 - Modelo Atualizado $\quad 37$

Figura 22 - Arquitetura do Framework Castor JDO [Castor05] 38

Figura 23 - Exemplo do Funcionamento do Framework Hibernate 
[Hibernate] 40

Figura 24 - Visão Geral do Framework iBatis [iBATIS] 41

Figura 25 - Camadas do Framework OJB [OJB05] 42

Figura 26 - Visão Geral do Processo Proposto 44

Figura 27 - Modelo PIM 46

Figura 28 - Mapeamento Marcação (Modelo) x Camada (Base de Conhecimento) $\quad 47$

Figura 29 - Base de Conhecimento 48

Figura 30 - Exemplificando a Base de Conhecimento 49

Figura 31 - Modelo PIM e seu XMI correspondente $\quad 50$

Figura 32 - Tags da Camada de Persistência $\quad 51$

Figura 33 - Arquivos RDL da Tag Hibernate 52

Figura 34 - Arquivo RDL utilizando o caracter "?" 53

Figura 35 - Exemplo de um Arquivo RDL 53

Figura 36 - Resultado da Transformação 54

Figura 37 - Modelo PSM gerado para o Framework de Persistência Castor 55

Figura 38 - Arquivo XML da configuração do banco para o Framework

Hibernate $\quad 56$

Figura 39 - Arquivo XML contendo o mapeamento tabela-classe do

Framework Hibernate 56

Figura 40 - Modelo PIM do Sistema de Sincronização dos Dados

Acadêmicos do SAU e do AulaNet $\quad 59$

Figura 41 - Modelo PIM Configurado para a Camada de Persistência 60

Figura 42 - Arquivo RDL do Padrão DAO 61

Figura 43 - Arquivo RDL do Padrão Facade 62

Figura 44 - Modelo PSM Gerado 63

Figura 45 - Comparação do Modelo Original com o Modelo Gerado pelo Processo 64

Figura 46 - Modelo PIM do Site de Leilão Virtual 66

Figura 47 - Modelo PIM Configurado para a Camada de Persistência 67

Figura 48 - Arquivo RDL que contêm as regras de transformações do framework de persistência Hibernate 68

Figura 49 - Modelo PSM Gerado através do Processo Proposto e Importado na Ferramenta CASE Poseidon 69

Figura 50 - Modelo da Camada de Persistência Finalizado pelo

PSM Designer $\quad 70$ 
Figura 51 - Visão Geral da Ferramenta $\quad 72$

Figura 52 - Funcionamento do Módulo $1 \quad 73$

Figura 53 - Modelo da Base de Conhecimento 73

Figura 54 - Janela de Manutenção de Camadas $\quad 74$

Figura 55 - Janela de Manutenção de Tags $\quad 75$

Figura 56 - Janela de Manutenção de Arquivos RDL 76

Figura 57 - Funcionamento do Módulo 2

Figura 58 - Janela de Importação do Arquivo XML Contendo o Modelo PIM 78

Figura 59 - Janela para Efetuar as Transformações (Escolha da Camada) 79

Figura 60 - Janela para Efetuar Transformações (Escolha da Tecnologia) 79

Figura 61 - Geração dos Arquivos XML de Configuração 80

Figura 62 - Janela de Exportação do Arquivo XMI Contendo o Modelo

PSM Gerado 